\title{
O TRABALHO EM EQUIPE NA ENFERMAGEM E OS LIMITES E POSSIBILIDADES DA ESTRATÉGIA SAÚDE DA FAMÍLIA
}

\author{
TEAMWORK IN NURSING AND THE LIMITS AND POSSIBILITIES OF THE FAMILY HEALTH STRATEGY \\ EL TRABAJO EN EQUIPO EN LA ENFERMERÍA Y LOS LÍMITES Y POSIBILIDADES DE LA ESTRATEGIA \\ SALUD DE LA FAMILIA
}

\author{
Maria de Lourdes Custódio Duarte ${ }^{1}$ \\ Jocemara Neves Boeck ${ }^{2}$
}

Resumo Este estudo buscou analisar a percepção dos profissionais de enfermagem sobre o trabalho em equipe em uma unidade da Estratégia Saúde da Família de um município do Sul do Brasil. Trata-se de uma pesquisa qualitativa, na qual se entrevistou a totalidade da equipe de enfermagem, compreendida por dois enfermeiros e seis técnicos de enfermagem. Com base na análise dos dados, emergiram duas categorias: limites encontrados para o trabalho em equipe; e possibilidades para um trabalho em equipe. Na primeira categoria evidenciaram-se algumas dificuldades, tais como gestão autoritária, entraves políticos, falta de motivação e reconhecimento e alta rotatividade de profissionais. Na segunda, os entrevistados sugeriram algumas possibilidades para o trabalho em equipe, como o conhecimento das habilidades de cada integrante da equipe, comunicação efetiva e reuniões de equipe. Conclui-se que os gestores de saúde devem aproximar-se mais dos trabalhadores e do cotidiano de trabalho dessas equipes, a fim de dar voz a esses profissionais, para promover a satisfação no trabalho e qualificar a assistência prestada à população.

Palavras-chave enfermagem; serviços de saúde; trabalhadores; atenção primária à saúde.
Abstract This study investigates the perception nursing professionals have about teamwork at a Family Health Strategy unit in a municipality in Southern Brazil. The entire nursing team, consisting of two nurses and six nursing technicians, was interviewed in this qualitative survey. Two categories emerged based on the analysis of the data: limits in teamwork, and possibilities for teamwork. The first category showed a few difficulties, such as authoritarian management, political barriers, lack of motivation and recognition, and high levels of staff turnover. In the second, respondents suggested a few possibilities for teamwork, such as knowledge about each team member's skills, effective communication, and team meetings. It is concluded that health managers should come closer to the workers and to the daily work done by these teams in order to give voice to these professionals, promote job satisfaction, and qualify the assistance provided to the population.

Keywords nursing; health services; workers; primary health care. 


\section{Introdução}

O trabalho em equipe é uma forma eficiente de estruturação, organização e aproveitamento das habilidades humanas. Possibilita uma visão mais global e coletiva do trabalho, reforça o compartilhamento de tarefas e a necessidade de cooperação para alcançar objetivos comuns. A concepção de trabalho em equipe está vinculada à de processo de trabalho e vem, portanto, sofrendo transformações ao longo do tempo, caracterizando-se pela relação recíproca entre as dimensões complementares de trabalho e interação (Pereira, Rivera e Artmann, 2013).

No campo de práticas da saúde, pensar o trabalho em equipe como uma imposição, uma diretriz do sistema, significa retrocesso sem igual. A construção do trabalho em equipe requer interação, comunicação e capacidade para colocar-se no lugar do outro, entendendo os diferentes saberes, em cada especificidade.

A ação interdisciplinar no trabalho em equipe, nesse contexto, pressupõe a possibilidade de a prática de um profissional se reconstruir mediante a prática do outro, ambos sendo transformados para a intervenção na realidade em que estão inseridos (Pereira, Rivera e Artmann, 2013; Scherer, Pires e Jean, 2013). Assim, é a soma dos diferentes saberes e conhecimentos de uma equipe multiprofissional que incidirá sobre os resultados da intervenção do processo saúde/doença, ou seja: a integralidade das ações de saúde está condicionada ao trabalho em equipe. No entanto, a integralidade só se realizará com incorporações ou redefinições mais radicais da equipe de saúde e de seus processos de trabalho (Martins et al., 2012).

$\mathrm{Na}$ área da saúde, obstáculos como a não cooperação, estruturas rigidamente hierarquizadas, a disparidade social entre os membros da equipe e a alta rotatividade dos profissionais nos setores são os principais entraves para a efetividade do trabalho integrado. Tais dificuldades devem ser trabalhadas para que não prejudiquem os usuários no acesso ao direito à saúde (Uchôa et al., 2012).

$\mathrm{O}$ interesse em desenvolver este trabalho deu-se a partir do ingresso de uma das autoras na Secretaria de Saúde e Assistência Social de um município do Sul do Brasil, onde se percebeu que as relações de trabalho na área da enfermagem de uma unidade da Estratégia Saúde da Família (ESF) não se configuravam como trabalho em equipe, dando a impressão de que eram pontos desconectados, quase rivalizados, e com um ambiente de competição. Tal observação nos levou a conhecer o funcionamento das equipes de saúde, sua composição e a relação com os gestores.

Nesse cenário, é importante entender o trabalho em equipe como integralidade, troca de conhecimento, união em busca do mesmo resultado, comprometimento com a saúde como um todo e principalmente sentimento 
de pertencimento à equipe. Não basta fazer parte 'da equipe' de trabalho; é necessário sentir a equipe e identificar-se com ela.

O trabalho em equipe parece ser algo tão simples e fácil de ser levado a termo, quase natural, já que os seres humanos são considerados gregários. Então, questiona-se: qual a percepção da equipe de enfermagem de uma unidade ESF sobre o trabalho em equipe? Portanto, tem-se por objetivo analisar a percepção da equipe de enfermagem sobre o trabalho em equipe em uma unidade da ESF.

\section{Método}

Este estudo teve caráter exploratório-descritivo, com abordagem qualitativa (Minayo, 2012). Foi realizado em um município de 19 mil habitantes do interior do estado do Rio Grande do Sul (Instituto Brasileiro de Geografia e Estatística, 2009). Os participantes compreenderam a totalidade da equipe de enfermagem de uma unidade da ESF do município: dois enfermeiros e seis técnicos de enfermagem, totalizando oito entrevistados. A escolha dos participantes foi intencional, por meio de convite, tendo como critério de inclusão: profissionais que estivessem em pleno exercício da função no período da coleta de dados e com vínculo empregatício de, no mínimo, seis meses; e como critério de exclusão: os que estivessem afastados do trabalho por motivo de licença-saúde ou férias. A coleta dos dados ocorreu em dezembro de 2011, por meio de entrevistas individuais semiestruturadas, consistindo de três indagações: para você, o que é trabalho em equipe? Quais os limites para que o trabalho seja realizado em equipe? Quais as possibilidades para melhorar o trabalho em equipe nesta unidade da ESF?

Procedeu-se à leitura integral das entrevistas, adotando-se o método de análise temática para apreciação crítica do conteúdo. Buscou-se encontrar os trechos significativos para a constituição dos temas abordados em obras pesquisadas em relação ao objetivo do estudo. Seguiram-se os passos de pré-análise e exploração do material, o que possibilitou a organização e a leitura repetida do corpus de pesquisa. Posteriormente, procedeu-se ao tratamento e interpretação dos resultados obtidos, descritos em unidades de registro e de contexto, que permitiram o agrupamento de ideias relevantes em categorias (Minayo, 2012), representadas por dois eixos temáticos de discussão: limites encontrados para o trabalho em equipe; e possibilidades para um trabalho em equipe.

Os participantes deste estudo foram numerados de 1 a 8 na ordem cronológica da realização das entrevistas e denominados de ' $E$ ' para os enfermeiros e ' $\mathrm{T}$ ' para técnicos de enfermagem. As entrevistas foram agendadas, e os dados, coletados em uma sala reservada no próprio local de trabalho dos profissionais, com tempo de duração em torno de trinta minutos. Os 
participantes assinaram o termo de consentimento livre e esclarecido após terem sido informados sobre os objetivos da pesquisa, aprovada pelo Comitê de Ética da Universidade Federal do Pampa, sob o parecer de n. 059/2011. Consideraram-se os aspectos éticos envolvendo seres humanos, em conformidade com o previsto na resolução n. 466/2012 do Conselho Nacional de Saúde (Brasil, 2012).

\section{Limites encontrados para o trabalho em equipe}

A proposição de mudança das práticas no Sistema Único de Saúde (SUS) pressupõe um saber-fazer comum, que contribui para a superação da fragmentação no cotidiano dos serviços. Não existe integralidade onde não haja troca de conhecimentos, característica fundamental dos serviços de saúde para a construção de práticas integrais (Uchôa et al., 2012). Assim, na presente categoria, foram verbalizadas pelos entrevistados algumas dificuldades para o trabalho em equipe: gestão autoritária; questões políticas; falta de motivação e reconhecimento; e alta rotatividade de profissionais.

Pode-se afirmar que uma gestão autoritária em um serviço de saúde é uma forma de gerenciar na qual não há consulta de opiniões dos profissionais em uma perspectiva tradicional. Esse tipo de gestão é um estilo mais diretivo, centrado na hierarquia e na burocracia (Garcia e Santa-Barbara, 2009). Embora, atualmente, esse tipo de gestão seja pouco adotado no setor de saúde, há ainda gestores que trabalham nessa lógica. Para um enfermeiro entrevistado, o autoritarismo da gestão é percebido quando os trabalhadores são valorizados apenas no momento em que produzem trabalho.

Na opinião do executivo, ele é quem manda: as ações em saúde são como esteiras e os funcionários são como máquinas, que só são aproveitadas enquanto produzem. Após, são descartadas... (E2).

A integralidade aponta para a ruptura de valores tradicionais na saúde, como hierarquia, distribuição de categorias profissionais e compartimentalização do saber (Moliner e Lopes, 2013). Nessa lógica, o trabalhador passa a ser percebido como um objeto, passível de descarte e de posterior reposição. Esse tipo de gestão influencia negativamente no trabalho em equipe na ESF, levando o trabalhador a uma desmotivação para a realização das suas atividades.

É atribuída ao gestor e sua forma de gerenciar o serviço de saúde a responsabilidade pela manutenção de um ambiente de trabalho saudável, por meio do estímulo de relações empáticas e harmoniosas entre os membros da equipe, o que certamente irá se refletir no cuidado prestado ao paciente (Balsanelli, Cunha e Whitaker, 2009). 
Em um município pequeno, as questões políticas parecem estar muito presentes no cotidiano da unidade da ESF estudada. Na fala de um técnico de enfermagem, verifica-se que estão imbricadas questões como desvalorização e sentimento de despertencimento que podem ter sido afloradas com o modo de gerenciar o trabalho em saúde dos gestores atuais.

O que atrapalha muito as ações para um verdadeiro trabalho em equipe são as atitudes políticas, que, por mais que pensem que somos 'burros', não somos (T1).

O modo de gerir a saúde influencia diretamente no trabalho em equipe de enfermagem da unidade da ESF estudada, tendo em vista que o profissional se sentia desqualificado pelas atitudes políticas dos gestores de saúde. Essa percepção vai de encontro aos atuais pressupostos de gestão compartilhada com ênfase em processos participativos, sendo entendida como um processo de fortalecimento de um grupo de trabalho, em que se ressaltam e se valorizam as competências individuais, diluindo o poder e fazendo com que cada membro reconheça o propósito e o significado de seu trabalho (Bonato, 2011).

Nesse cenário, entende-se que a gestão autoritária e as questões políticas adotadas no município estudado refletem a falta de reconhecimento da equipe, repercutindo na desmotivação dos trabalhadores da enfermagem. Dessa maneira, a motivação pode ser entendida como um fator que impulsiona as pessoas a fazerem algo que pode ser desde o suprimento das necessidades humanas básicas até a autorrealização profissional (Bendassolli, 2012).

Falta motivação para as equipes, os salários também (...) deixam a desejar (T3).

Uma equipe pode trabalhar com poucos recursos materiais, mas tem que se sentir valorizada pela sua chefia. Se tu sentires que és reconhecido pelo teu trabalho, que tu és importante naquela função, é diferente o resultado (...) (E4).

Falta o reconhecimento por parte da chefia (...) a ordem é dada sem perguntarem a tua opinião (...) Num dia tu chegas e simplesmente tu vais ser trocado de equipe e pronto, tu tens que ir (...) e o trabalho que tu estavas desenvolvendo lá, não tem importância nenhuma? (...) (T5).

Eu só faço meu feijão com arroz (...) Não adianta tu quereres fazer diferente, tu não és valorizado, não tem incentivo nenhum (T6).

Os profissionais entendiam que existem alguns elementos motivadores para a construção do trabalho em equipe e que estão relacionados diretamente à sua valorização e reconhecimento como profissionais, como questão 
salarial, falta de recursos materiais e valorização do trabalho realizado. Dessa maneira, na gestão de pessoas, o reconhecimento é amiúde tratado como elemento-chave da relação do sujeito com o trabalho e a organização, com implicações diretas nos processos motivacionais e nas percepções de valorização do trabalhador e de justiça. Neste último sentido, o reconhecimento está associado às expectativas de retribuição pela contribuição aportada pelos indivíduos à organização (Lima et al., 2014).

O reconhecimento é também apontado como nuclear em processos de construção identitária de saúde e prazer no trabalho. Assim, o reconhecimento pode ser entendido como um processo de retribuição simbólica assentado em julgamentos sobre o fazer das pessoas. A psicodinâmica tem chamado a atenção para o nexo entre falta de reconhecimento e processos de sofrimento e adoecimento. Inversamente, tem mostrado a importância do reconhecimento nos processos de construção de significados, mediando a relação do sujeito com o outro no contexto de trabalho, inscrevendo-o numa história coletiva e em circuitos de utilidade (valor) e beleza (qualidade) (Merlo e Mendes, 2009). A falta de incentivo por parte dos gestores em saúde gerava desânimo e o não reconhecimento pelas atividades desenvolvidas na ESF, fazendo com que os profissionais entrevistados desenvolvessem ações e tarefas restritas ao seu fazer cotidiano. Logo, a não valorização da equipe de enfermagem e o não reconhecimento desse trabalho promovem uma desintegração da equipe como um todo, desfazendo o fazer coletivo.

O sentimento de não pertença de um grupo, gerado por todos os fatores até aqui expostos, provocava uma alta rotatividade dos profissionais e uma sensação persistente de um constante recomeçar.

Às vezes, quando a gente tá começando a engrenar o trabalho em equipe, a chefia vem e troca alguém de ESF (...) Isto faz com que a gente tenha que começar tudo de novo, se perde tempo, motivação (E4).

Evidencia-se que as trocas de membros da equipe de enfermagem estudada eram realizadas sem consultar os profissionais envolvidos. Esse processo de transferência involuntária gerava insatisfação para os que eram transferidos e também para a equipe que permanecia na unidade da ESF.

Dessa forma, recomenda-se que os gestores criem estratégias e rotinas para reconhecer os profissionais, motivando-os para o trabalho em equipe. Consultar os profissionais antes de tomar decisão e buscar compreender as necessidades de cada trabalhador são atitudes que podem influenciar na atuação de cada trabalhador e, consequentemente, no trabalho em equipe (Martins et al., 2012). Por fim, recomenda-se aos gestores desenvolverem habilidades de dar e receber feedback periodicamente ao trabalhador, proporcionando um espaço de fala e escuta. 
Portanto, a presente categoria evidencia que não é suficiente os trabalhadores interagirem cordialmente ou compartilharem uma mesma situação de trabalho para constituírem uma equipe integrada. É necessário um investimento na articulação das ações, preservando as especificidades de cada componente da equipe. Essa atitude requer por parte dos gestores de saúde o reconhecimento do trabalho do outro, pressupondo uma concepção ampla do processo saúde/doença no contexto da enfermagem.

\section{Possibilidades para o trabalho em equipe}

A possibilidade de um trabalho em equipe no setor de saúde é real e de extrema importância, tendo em vista a complexidade que se configura nos atuais problemas de saúde da população como um todo. Assim, na presente categoria, surgiram algumas possibilidades visualizadas pela equipe de enfermagem da unidade da ESF estudada para o trabalho em equipe: conhecimento das habilidades de cada integrante da equipe; comunicação efetiva; e reuniões de equipe.

O conhecimento tanto dos gestores quanto dos próprios integrantes da equipe de enfermagem sobre as habilidades e afinidades de cada trabalhador é importante para que o trabalho em equipe se efetive na prática do serviço. Assim, cada profissional trabalhará com mais prazer e eficiência.

Eu acho importante conhecer as habilidades de cada um e em qual setor cada um teria maior afinidade. Assim, todos trabalhariam por prazer (T1).

Acredito que o primeiro passo para a construção de uma equipe de sucesso é você conhecer cada um. Se você conhecer o potencial de cada um, vai saber até como falar com ele para que a conversa seja mais positiva ou construtiva, sem 'lesionar' a integridade de ambos (...) (E4).

O trabalho em equipe destaca-se no conjunto de características da ESF como um dos pressupostos mais importantes para a reorganização do processo de trabalho como possibilidade de uma abordagem mais completa (Bornstein e David, 2014). Logo, a interação e o conhecimento entre os membros da equipe da ESF são necessários para que todos possam respeitar as individualidades de cada um dentro do seu núcleo de saber.

O profissional de enfermagem é formado para trabalhar em vários espaços e reconhecer de maneira mais aprofundada cada integrante da equipe, o que possibilita identificar afinidades com um ou outro setor. Assim, a tendência não é a acomodação do profissional, e sim a busca pelo conhecimento dentro de uma área que lhe seja agradável. Nessa compreensão, o trabalho é 
fundamental para o homem, pois por meio dele atinge sua realização profissional, afirma-se como um ser social, um ser capaz de dar respostas práticas e conscientes às suas necessidades (Lima et al., 2013).

Nesse sentido, a comunicação é entendida como um fator indispensável para o trabalho em grupo na unidade da ESF estudada. Assim, o exercício do diálogo e de trocas no ambiente de trabalho facilita o trabalho em grupo, abrindo possibilidades para um trabalho resolutivo e comprometido com as necessidades da população.

O trabalho em equipe deve ser construído através da participação de todos aproveitando o que cada um tem de forte (...) na individualidade de cada um, quando se consegue juntar o que cada um faz de melhor dentro de suas especificidades (S1).

A chave é a comunicação. Poder dizer quando não conseguirá fazer a sua parte e pedir ajuda ao colega; dizer que precisa de suporte é fundamental para fluir o trabalho e este ser resolutivo (...) (S2).

Para o trabalho em saúde, é necessário pensar na unidade dos saberes, no respeito às especificidades das profissões da saúde e, principalmente, apostar no diálogo entre os profissionais, pois por meio da ação comunicativa pode-se construir novas formas de trabalho em saúde. Entretanto, a comunicação ineficaz traz desgastes nas relações, agressões verbais, mal-entendidos, suscetibilidades afetadas, perda de motivação e estresse (Chiavenato, 2006). Logo, é fundamental que os gestores de saúde reconheçam o papel da comunicação como uma estratégia para que o trabalho em equipe seja efetivo.

Quando indagados sobre sugestões para a construção de um trabalho em equipe na unidade da ESF estudada, os entrevistados citaram a criação de espaços de troca em reuniões. Tais espaços serviriam como um momento de integração, de escuta, de valorização do profissional e também de planejamento de trabalho.

Estipularia um momento de reunião no trabalho onde pudéssemos ter um momento de integração, de escuta da equipe, onde poderíamos explorar conceitos, os planos e mudanças (S6).

Promover reuniões de equipe e dar oportunidade para que todos possam falar e também serem ouvidos é muito importante e valoriza o trabalho profissional, sem contar no dia a dia... (S3).

A reunião de equipe é um instrumento importante para se gerenciar o serviço de saúde e fazer o planejamento das próximas ações (Kawata et al., 2009). 
No entanto, os entrevistados deste estudo foram além da concepção gerencial das reuniões. Entendiam esses momentos como espaços propícios para escutar e ouvir, dar e receber feedback sobre determinadas ações, visando à integração entre as pessoas da equipe, influenciando positivamente na comunicação e na interação entre esses agentes de saúde. Tais falas demonstram o quanto a equipe de enfermagem precisa de um espaço de escuta, de voz ativa e de interação entre as pessoas que compõem essa equipe na ESF. Portanto, nessa categoria foram discutidas as sugestões dos profissionais de enfermagem para um trabalho em equipe na unidade da ESF estudada.

\section{Considerações finais}

A discussão sobre o trabalho em equipe na enfermagem possibilitou escutar esses profissionais e identificar sua compreensão sobre os limites de trabalhar em equipe e as possibilidades para melhorar esse trabalho em uma unidade da ESF de um município de pequeno porte do Rio Grande do Sul.

A primeira categoria evidenciou as dificuldades enfrentadas pelos profissionais da enfermagem da unidade da ESF estudada. A gestão autoritária, questões políticas, falta de motivação e reconhecimento e alta rotatividade de profissionais foram aspectos verbalizados como agravantes que dificultavam o trabalho no serviço, prejudicando o trabalho em equipe.

Assim, entende-se que os profissionais que ali trabalhavam não realizavam suas atividades com prazer se fossem obrigados a fazê-las, se o convite fosse coercitivo, se não houvesse interesse ou afinidade pelo que estavam realizando. Faz parte do ser humano a necessidade de ser motivado a buscar algo novo, e com os trabalhadores da área da saúde isso não é diferente. A relação do profissional versus gestor deve ser reavaliada. Com base no diálogo e na escuta sensível de ambas as partes, é preciso encontrar um novo jeito de caminhar.

No entanto, os entrevistados demonstraram por meio de sugestões aspectos que poderiam melhorar o trabalho em equipe. As principais possibilidades verbalizadas pelos entrevistados foram a necessidade de o gestor de saúde e os próprios profissionais conhecerem as habilidades de cada integrante da equipe, melhorarem a comunicação entre os profissionais e instituírem com frequência reuniões que propiciassem espaços de fala e de escuta.

Trabalho em equipe não se faz sozinho, é uma construção em que todos os participantes devem interagir, expor seus anseios e habilidades, capacidades e dificuldades em grupo, canalizando-os para uma melhora na qualidade do trabalho da equipe de enfermagem na unidade da ESF estudada. Nessa perspectiva, entendemos que os profissionais de enfermagem têm intencionalidade na construção do trabalho em equipe e são comprometidos 
com o seu projeto profissional. As possibilidades de implementação do trabalho em equipe no município existem - e foram os próprios profissionais que apontaram as sugestões para a construção dessa competência.

Portanto, os resultados deste estudo evidenciam os limites e possibilidades dos profissionais de enfermagem de uma unidade da ESF para o trabalho em equipe. Esses dados indicam que os gestores de saúde devem aproximar-se mais dos trabalhadores e do cotidiano de trabalho dessas equipes, a fim de dar voz a esses profissionais, promovendo a satisfação no trabalho e qualificando a assistência prestada à população.

\section{Colaboradores}

Maria de Lourdes Custódio Duarte participou de todo o processo de elaboração do artigo. Jocemara Neves Boeck participou do processo de coleta de dados e análise.

Resumen Este estudio buscó analizar la percepción de los profesionales de enfermería sobre el trabajo en equipo en una unidad de la Estrategia Salud de la Familia de un municipio del Sur de Brasil. Se trata de una investigación cualitativa, en la cual se entrevistó la totalidad del equipo de enfermería, compuesta por dos enfermeros y seis técnicos de enfermería. En base al análisis de los datos, surgieron dos categorías: límites encontrados para el trabajo en equipo; y posibilidades para un trabajo en equipo. En la primera categoría se pusieron de manifiesto algunas dificultades, tales como gestión autoritaria, falta de motivación y reconocimiento, y alta rotación de profesionales. En la segunda, los entrevistados sugirieron algunas posibilidades para el trabajo en equipo, como el conocimiento de las habilidades de cada integrante del equipo, comunicación efectiva y reuniones de equipo. Se concluye que los gestores de salud deben aproximarse más a los trabajadores y al cotidiano quehacer de estos equipos, a fin de dar voz a estos profesionales, para promover la satisfacción en el trabajo y calificar la asistencia prestada a la población.

Palabras clave enfermería; servicios de salud; trabajadores; atención primaria a la salud. 


\section{Notas}

1 Universidade Federal do Rio Grande do Sul, Escola de Enfermagem, Porto Alegre, Rio Grande do Sul, Brasil.

$<$ malulcd@yahoo.com.br>

Correspondência: Rua Gonçalves Ledo, n. 20, apto. 203, bairro Partenon, CEP 90610-250, Porto Alegre, Rio Grande do Sul, Brasil.

2 Escola de Saúde Pública do Rio Grande do Sul, Porto Alegre, Rio Grande do Sul, Brasil. $<$ maranv@ibest.com.br>

\section{Referências}

BALSANELLI, Alexandre P.; CUNHA, Isabel C. K. O. C.; WHITAKER, Iveth Y. Estilos de liderança de enfermeiros em unidade de terapia intensiva: associação com perfil pessoal, profissional e carga de trabalho. Revista Latino-Americana de Enfermagem, Ribeirão Preto, v. 17, n. 1, p. 28-33, 2009.

BENDASSOLLI, Pedro. F. Reconhecimento no trabalho: perspectivas e questões contemporâneas. Psicologia em Estudo, Maringá, v. 17, n. 1, p. 37-46, 2012.

BONATO, Vera. L. Gestão de qualidade em saúde: melhorando assistência ao cliente. O Mundo da Saúde, São Paulo, v. 35, n. 5, p. 319-331, 2011.

BORNSTEIN, Vera J.; DAVID, Helena M. S. L. Contribuições da formação técnica do agente comunitário de saúde para o desenvolvimento do trabalho da equipe saúde da família. Trabalho, Educação e Saúde, Rio de Janeiro, v. 12, n. 1, p. 107-128, 2014.

BRASIL. Ministério da Saúde. Conselho Nacional de Saúde. Resolução n. 466, de 12 de dezembro de 2012. Aprova as diretrizes e normas regulamentadoras de pesquisas envolvendo seres humanos. Brasília: Ministério da Saúde, 2012. Disponível em: <http://conselho. saude.gov.br/resolucoes/2012/Reso466.pdf>. Acesso em: 5 dez. 2012.
CHIAVENATO, Idalberto. Recursos humanos: o capital humano das organizações. 8. ed. São Paulo: Atlas, 2006

GARCIA, Garcia. I.; SANTA-BARBARA, Emílio. S. Relação entre estilos de liderança e bases de poder das enfermeiras. Revista Latino-Americana de Enfermagem, Ribeirão Preto, v. 17, n. 3, p. 295-301, 2009.

INSTITUTO BRASILEIRO DE GEOGRAFIA E ESTATÍSTICA (IBGE). Censo 2009: estimativas das populações residentes, em $1^{\circ}$ de julho de 2009, segundo municípios. Rio de Janeiro, 2009. Disponível em: <www. ibge.gov.br/home/estatistica/populacao/ estimativa2009/POP2009_DOU.pdf $>$. Acesso em: 2 nov. 2010.

KAWATA, Lauren. S. et al. O trabalho cotidiano da enfermeira na saúde da família: utilização de ferramentas da gestão. Texto \& Contexto Enfermagem, Florianópolis, v. 18, n. 2, p. 313-320, 2009.

LIMA, Fabiano. B. et al. Fatores de motivação no trabalho de enfermagem. Revista de Pesquisa: Cuidado É Fundamental Online, Rio de Janeiro, v. 5, n. 4, p. 417-423, 2013. Disponível em: <www.seer.unirio.br/index. php/cuidadofundamental/article/view/3072>. Acesso em: 2 nov. 2011. 
LIMA, Letícia et al. Satisfação e insatisfação no trabalho de profissionais de saúde da atenção básica. Escola Anna Nery Revista de Enfermagem, Rio de Janeiro, v. 18, n. 1, p. 17-24, 2014.

MARTINS, Alexandra R. et al. Relações interpessoais, equipe de trabalho e seus reflexos na atenção básica. Revista Brasileira de Educação Médica, Rio de Janeiro, v. 36, n. 2, p. 6-12, 2012.

MERLO, Álvaro R. C.; MENDES, Ana M. B. Perspectivas do uso da psicodinâmica do trabalho no Brasil: teoria, pesquisa e ação. Cadernos de Psicologia Social do Trabalho, São Paulo, v. 12, n. 2, p. 141-156, 2009.

MINAYO, Maria C. S. O desafio do conhecimento: pesquisa qualitativa em saúde. 12. ed. São Paulo: Hucitec, 2012.

MOLINER, Jonas; LOPES, Stella M. B. Saúde mental na atenção básica: possibilidades para uma prática voltada para a ampliação e integralidade da saúde mental. Saúde e Sociedade, São Paulo, v. 22, n. 4, p. 1.072-1.083, 2013.
PEREIRA, Renata C. A.; RIVERA, Francisco. J. U.; ARTMANN, Elizabeth. The multidisciplinary work in the family health strategy: a study on ways of teams. Interface: Comunicação, Saúde, Educação, Botucatu, v. 17, n. 45 , p. 327-340, abr./jun. 2013.

SCHERER, Magda D. A.; PIRES, Denise. E. P.; JEAN, Rémy. A construção da interdisciplinaridade no trabalho da equipe de saúde da família. Ciência \& Saúde Coletiva, Rio de Janeiro, v. 18, n. 11, p. 3.203-3.212, 2013.

UCHÔA, Alice C. et al. Trabalho em equipe no contexto da reabilitação infantil. Physis: Revista de Saúde Coletiva, Rio de Janeiro, v. 22, n. 1, p. 385-400, 2012.

Recebido em 25/02/2013

Aprovado em 02/07/2014 